\title{
Coocurrencia de trastornos de personalidad en pacientes con trastornos de ansiedad
}

\section{Co-occurrence of Personality Disorders in Patients with Anxiety Disorders}

\author{
Humbelina Robles-Ortega ${ }^{\mathrm{a}, \mathrm{b}}$, José Luis Mata-Martín ${ }^{\mathrm{a}, \mathrm{b}}$, María Blasa Sánchez-Barrera ${ }^{\mathrm{a}, \mathrm{b}}$, María Nieves Vera- \\ Guerrero $^{\mathrm{a}, \mathrm{b}}$, Joaquín Pegalajar ${ }^{\mathrm{a}, \mathrm{b}}$, Andrea Mezcua ${ }^{\mathrm{c}}$, María Carmen Fernández-Santaella ${ }^{\mathrm{a}, \mathrm{b}}$, \& Jaime Vila ${ }^{\mathrm{a}, \mathrm{b}}$ \\ ${ }^{a}$ Universidad de Granada, Granada, España ${ }^{b}$ Centro de Investigación Mente, Cerebro y Comporta- \\ miento, Granada, España ${ }^{c}$ Granada, España
}

\begin{abstract}
Resumen: La coocurrencia entre los trastornos de personalidad y los trastornos de ansiedad puede tener implicaciones importantes. Los diagnósticos múltiples nos pueden dar idea de la gravedad de la alteración, el deterioro del funcionamiento y el pronóstico. El objetivo de este estudio fue doble, analizar la coocurrencia entre trastornos de personalidad y trastornos de ansiedad, e identificar perfiles de trastornos de personalidad en los diferentes trastornos de ansiedad. En este estudio participaron 31 pacientes con trastornos de ansiedad, distribuidos en cuatro grupos: fobia específica, fobia social, ansiedad generalizada y pánico, con y sin presencia de agorafobia. Los instrumentos de evaluación utilizados fueron El Inventario Clínico Multiaxial de Millon II y la Entrevista para los Trastornos de Ansiedad según el Manual diagnóstico y estadístico de los trastornos mentales IV. El grupo de fobia específica presenta puntuaciones más bajas en las distintas escalas y menor frecuencia de trastornos de personalidad. Por el contrario, el grupo de ansiedad generalizada presenta mayor tasa de diagnósticos múltiples de trastornos de personalidad. Las puntuaciones más altas se observan en las escalas esquizoide, dependiente, autodestructiva, evitativa y compulsiva. Cada trastorno de ansiedad presenta un perfil de trastornos de personalidad. Tener en cuenta la coocurrencia entre trastornos puede ayudar a comprender el éxito o no de un tratamiento, por lo que la evaluación sistemática de la personalidad patológica en el ámbito clínico es recomendable.
\end{abstract}

Palabras clave: coocurrencia, trastornos de ansiedad, trastornos de personalidad.
Abstract: The co-occurrence between personality disorders and anxiety disorders may have important implications. Multiple diagnoses can give us an idea of the severity of the disorder, functioning impairment and prognosis. The aim of this study was two-fold, to analyze the co-occurrence between personality disorders and anxiety disorders, and to identify personality disorder profiles in different anxiety disorders. A total of 31 patients with anxiety disorders participated in the study. They were divided into four groups: specific phobia, social phobia, generalized anxiety and panic with or without the presence of agoraphobia. The assessment instruments used were The Millon Clinical Multiaxial Inventory II and the Anxiety Disorders Interview according to the fourth edition of the Diagnostic and Statistical Manual of Mental Disorders. The specific phobia group presented lower scores on the different scales and lower frequency of personality disorders. On the contrary, the generalized anxiety group had a higher rate of multiple diagnoses of personality disorders. Higher scores were observed in the schizoid, dependent, self-defeating, avoidant and compulsive scales. Each anxiety disorder presented a profile of personality disorders. Co-occurrence between disorders may help to understand the success or failure of a treatment, so the systematic evaluation of pathological personality in the clinical setting is recommended.

Keywords: co-occurrence, anxiety disorders, personality disorders.

Contacto: H. Robles-Ortega. Depto. de Personalidad, Evaluación y Tratamiento Psicológico, Facultad de Psicología, Universidad de Granada, Campus de Cartuja s/n 18071, Granada, España. Correo electrónico: hrobles@ugr.es

Cómo citar: Robles-Ortega, H., Mata-Martín, J. L., Sánchez-Barrera, M. B., Vera-Guerrero, M. N., Pegalajar, J., Mezcua, A., ... Vila, J. (2016). Coocurrencia de trastornos de personalidad en pacientes con trastornos de ansiedad. Revista de Psicología, 25(1), 1-17.

http://dx.doi.org/10.5354/0719-0581.2016.41466 


\section{Introducción}

A pesar de conocerse la importancia de los trastornos de la personalidad (TP) desde hace mucho tiempo, es desde hace poco que se han convertido en un tema de gran relevancia para la investigación. La inclusión de los TP en el Manual diagnóstico y estadístico de los trastornos mentales (DSM, por su nombre en inglés Diagnostic and Statistical Manual of Mental Disorders; American Psychiatric Association [APA], 1980), constituyó un hito importante, tanto en el ámbito clínico como en el ámbito de la investigación. A pesar de los años transcurridos, sigue siendo un campo bastante desconocido, por lo que se puede aseverar que los TP constituyen el gran reto del siglo XXI (Gunderson \& Gabbard, 2000).

Los TP son definidos como un patrón permanente de conducta y de experiencia interna que se aparta acusadamente de las expectativas de la cultura del individuo; este patrón se manifiesta en algunas de las siguientes áreas: cognición, afectividad, funcionamiento interpersonal y control de impulsos (APA, 2013). Los TP son clasificados en tres grandes grupos: grupo A (sujetos extraños o excéntricos), grupo B (sujetos teatral-emotivos e inmaduros) y grupo C (sujetos ansioso-temerosos) (APA, 2013).

Uno de los aspectos de los TP que tiene un gran interés en el campo de la psicopatología, es la comorbilidad (Jakovljević \& Crnčević, 2012). Aunque muchos investigadores y clínicos utilizan dicho término, lo cierto es que es un concepto problemático. En los últimos años, se van levantando voces contrarias a su utilización dentro del ámbito psiquiátrico, argumentando que se trataría de una anomalía conceptual; dado que este término hace referencia a la presencia de dos o más diagnósticos psiquiátricos que ocurren juntos, pero en la realidad no está tan claro que se trate de dos condiciones clínicas diferentes, sino que podría tratarse de múltiples manifestaciones de una sola condición clínica (Aragona, 2009; Jakovljević \& Crnčević, 2012). Por ello, se va a utilizar el término coocurrencia.

La coocurrencia de los TP, del eje II del DSM IV (APA, 1994) con otros síndromes clínicos (recogidos en el Eje I) se ha constatado en diversos estudios, que van desde el consumo de cocaína (López Durán \& Becoña Iglesias, 2006), trastornos del estado de ánimo (Dyck, et al., 2001; Prieto Cuéllar, Vera Guerrero, Pérez Marfil, \& Ramírez Uclés, 2007), juego patológico (Fernández Montalvo \& Echeburúa, 2006), trastorno bipolar (Dunayevich et al., 2000), trastornos de ansiedad (TA) (Flick, Roy-Byrne, Cowley, Shores, \& Dunner, 1993; Markovitz, 2004; Reich, 2009; Zimmerman, Rothschild, \& Chelminski, 2005) y personas sin hogar (Salavera, Puyuelo, Tricás, \& Lucha, 2009).

Se cree que es de gran relevancia conocer el efecto que los TP pueden tener en los síndromes clínicos y el valor de pronóstico de dicha coocurrencia. En este contexto, poco a poco se van publicando más estudios, pero los resultados no siempre son consistentes. Diversos estudios señalan que la coocurrencia de los TP, con otros síndromes clínicos del eje I, apunta a un empeoramiento del cuadro clínico, peor adherencia y respuesta al tratamiento, mayor riesgo de recaídas y hospitalización, peor pronóstico y mayor nivel de psicopatología general (Dunayevich et al., 2000; Friborg, Martinussen, Kaiser, Øvergård, \& Rosenvinge, 2013; NewtonHowes et al., 2010; Ozkan \& Altindag, 2005; Reich, 2009; Rossi et al., 2001; 
Sandoval \& Villamil, 1999; Telch, Kamphuis, \& Schmidt, 2011). Los costos de salud indican una carga económica al sistema de atención de la salud marcadamente superior (Soeteman, Hakkaart-van Roijen, Verheul, \& Busschbach, 2008). En esta misma línea, el tratamiento de los TP coocurrentes es normalmente más complejo y menos optimista, con un aumento de las tasas de abandono y con menos expectativas de los pacientes (Martino, Menchetti, Pozzi, \& Berardi, 2012; Reich, 2009; Sanderson, Beck, \& McGinn, 1994; Telch et al., 2011).

Sobre la relación existente entre los TP y los TA (trastornos de pánico con o sin agorafobia, fobia social y trastornos de ansiedad generalizada), se han establecido algunas hipótesis en las que los TP constituyen un riesgo para el desarrollo de trastorno de pánico y trastorno de pánico con agorafobia (Brown \& Barlow, 2009; Kotov, Gámez, Schmidt, \& Watson, 2010). Así, por ejemplo, Bienvenu et al. (2009) encontró en su investigación que puntuaciones altas en las escalas de evitación y dependencia (trastornos del grupo C) predicen la aparición de trastorno de pánico y agorafobia.

En esta misma línea, un estudio de metanálisis basado en 125 estudios publicados en los últimos treinta años (Friborg et al., 2013) concluye que en muestras de TA, en general, se observa en mayor proporción coocurrencia con trastornos del grupo $\mathrm{C}$ (evitación, dependencia y obsesivo-compulsivo) que con trastornos de los grupos A y B. Y dentro del grupo $\mathrm{C}$, el patrón de mayor coocurrencia fue el evitativo, seguido del obsesivo y del dependiente.

Los perfiles de TP más frecuentemente encontrados en muestras de personas con diagnóstico de trastorno de pánico y ago- rafobia, han sido la personalidad evitativa, dependiente y obsesivo-compulsiva (Albert, Maina, Bergessio, \& Bogetto, 2006; Marchesi et al., 2006; Osma, García-Palacios, Botella, \& Barrada, 2014a, 2014b; Starcevic et al., 2008; Telch et al., 2011).

El trastorno de pánico y agorafobia es uno de los más abordados por la investigación en el campo de la coocurrencia con los TP (Albert et al., 2006; Marchesi et al., 2006; Marchesi, Cantoni, Fontò, Giannelli, \& Maggini, 2005; Osma et al., 2014a, 2014b). En cambio, no existen tantos estudios que aborden la coocurrencia de los TP y el resto de los TA.

Entre los pocos estudios publicados, se puede citar el estudio de Stinson et al. (2007), que encontró en mujeres con TP narcisista una asociación con trastorno de ansiedad generalizada y trastorno bipolar; en cambio, en hombres, el cuadro narcisista se asoció con el consumo de sustancias. Otro estudio que se puede citar es el de Flick et al. (1993), que encontró que los pacientes ambulatorios con diferentes cuadros de ansiedad, sufrían TP de tipo evitativo, paranoide, límite y obsesivocompulsivo. A esta misma coocurrencia se refería Markovitz (2004) en personas mayores.

Autores como Reich (2009) corroboran esta misma situación en una investigación realizada en pacientes con cuadros de ansiedad (pánico, ansiedad generalizada, agorafobia y fobia social). La relación más alta se daba con la fobia social y con el trastorno de ansiedad generalizada.

El efecto del TP por evitación coocurrente con el diagnóstico de fobia social, se ha podido observar en la respuesta al tratamiento para este trastorno en pacientes ambulatorios con diagnóstico de fo- 
bia social generalizada. Los pacientes que presentaban coocurrencia estuvieron más gravemente afectados en todas las medidas de autoinforme en el pretest. Y, aunque después del tratamiento los pacientes con fobia social mejoraron significativamente, los que presentaban coocurrencia con TP siguieron mostrando un deterioro más grave en las medidas postratamiento y después de 3 meses de seguimiento, en comparación con aquellos que no presentaban coocurrencia (Feske, Perry, Chambless, Renneberg, \& Goldstein, 1996).

Otros estudios han encontrado una alta asociación de la personalidad por evitación con depresión mayor, agorafobia, fobia social y trastorno obsesivo-compulsivo (Zimmerman et al., 2005).

Por otra parte, también se ha examinado la relación de los TP y los TA, teniendo en cuenta el efecto de la depresión mayor. El 24\% de los participantes tenía al menos un TP, como la personalidad evitativa, obsesivo-compulsiva o dependiente. El trastorno de ansiedad generalizada, fobia social y depresión mayor, se asoció positivamente con uno o más trastornos de la personalidad, mientras que no se observó esta asociación con el trastorno de pánico con agorafobia (Dyck et al., 2001). Estudios más recientes han encontrado asociación entre la fobia social y los TP por evitación y dependencia (Friborg et al., 2013; Grant et al., 2005).

Con respecto al trastorno de fobia específica, no existen demasiados estudios que aborden la coocurrencia de este trastorno con los TP. Es posible que se deba a que este tipo de trastorno, comparado con el resto de los TA, es uno de los que menos incapacitación produce, debido a que el objeto fóbico es muy específico y localizado (Belloch, Sandín, \& Ramos, 2008).

Sin embargo, no todos los casos de fobias específicas son tan localizados. Algunos estudios indican que dentro de este trastorno también podemos encontrar una gran variabilidad. Así, por ejemplo, Stinson et al. (2007) encontraron que en muchos casos más generalizados, las fobias conllevan múltiples miedos, mayor discapacidad y deterioro, búsqueda de tratamiento y una mayor coocurrencia, con otros trastornos del eje I y II. Al igual que en la fobia social, estos casos reflejan una forma generalizada de fobia específica.

Otros TP, como es el caso del trastorno límite de la personalidad, han sido comúnmente asociados a otro tipo de problemas, como depresión mayor, dependencia de sustancias, dependencia del alcohol y trastorno de déficit de atención con hiperactividad (Wetterborg, Långström, Andersson, \& Enebrink, 2015).

Un aspecto de gran relevancia es el referido al diagnóstico múltiple de los TP. Es bastante frecuente que en una misma persona se observen varios TP (Dyck et al., 2001; Flick et al., 1993; Salavera et al., 2009). Se ha propuesto que el diagnóstico múltiple de TP, tiene implicaciones importantes.

El número de diagnósticos por sujeto refleja la gravedad de la alteración en este, ya que se correlaciona con la severidad de la psicopatología y el deterioro del funcionamiento (Dolan, Evan, \& Norton, 1995; Oldham et al., 1992; Salavera et al., 2009). El diagnóstico múltiple de TP (coexistencia de dos o más TP en la misma persona) dificulta su proceso de recuperación, empeorando su pronóstico y com 
plicando la interpretación de los resultados (Dolan et al., 1995; Salavera et al., 2009).

No cabe duda de que uno de los aspectos que más preocupa al clínico, son las variables que están implicadas en la dificultad de recuperación de los pacientes tratados (Dunayevich et al., 2000; Martino et al., 2012; Reich, 2003; Sanderson et al., 1994; Telch et al., 2011). ¿Qué variables influyen? ¿Por qué un tratamiento no es igualmente efectivo para todos los pacientes que lo reciben? Una de las explicaciones podría estar en la coocurrencia con los TP. Por ello, el objetivo de este de este estudio -de tipo descriptivo- es explorar la coocurrencia entre los TP y los TA. Es de interés determinar, por una parte, si hay perfiles específicos de personalidad patológica en los distintos TA evaluados (primer objetivo) $\mathrm{y}$, por otra parte, si el número de TP en los pacientes (indicativo de gravedad) es diferente en función del TA diagnosticado (segundo objetivo).

\section{Método}

\section{Participantes}

En el presente estudio participaron 31 pacientes de la Clínica de Psicología de la Facultad de Psicología de la Universidad de Granada, 5 hombres y 26 mujeres, distribuidos en cuatro grupos, en función del diagnóstico recibido: fobia específica $(n=9)$, fobia social $(n=4)$, trastornos de ansiedad generalizada $(n=6)$ y trastorno de pánico y agorafobia $(n=12)$.

Todos los participantes en este estudio habían solicitado ayuda por sus problemas de ansiedad en la Clínica de Psicología. El sesgo en la muestra (mayoritariamente mujeres) refleja una realidad observada en la Clínica: un porcentaje muy alto de usuarios de estos servicios son mujeres.

La edad media de los participantes fue de 28,80 años $(D T=9,36)$, no existiendo diferencias significativas entre los cuatro grupos en esta variable $(p=, 48)$. Todos ellos responden al perfil de estudiantes universitarios $(n=17)$, personal de administración y servicios, o personal docente e investigador de la Universidad de Granada $(n=14)$.

Los criterios de inclusión en este estudio, fueron ser mayor de 18 años y recibir un diagnóstico principal de TA en la Clínica de Psicología; entendiendo por diagnóstico principal aquel por el que la persona solicita ayuda psicológica, siguiendo los planteamientos del DSM IV (APA, 1994). Los criterios de exclusión fueron presentar otro síndrome clínico (como por ejemplo, trastorno del estado de ánimo, trastorno de la conducta alimentaria) coocurrente con el diagnóstico principal de TA.

\section{Instrumentos de evaluación}

Se aplicaron dos instrumentos de evaluación. El primero es el Inventario Clínico Multiaxial de Millon II (MCMI-II, por su nombre en inglés Millon Clinical Multiaxial Inventory; Millon, 1999), versión española; autoinforme ampliamente utilizado para el estudio de la personalidad y sus trastornos en personas de población clínica.

Este instrumento consta de 175 ítems con dos opciones de respuesta (verdadero o falso), agrupados en 22 escalas, de las cuales 10 son escalas básicas de personalidad (esquizoide, evitativa, dependiente, histriónica, narcisista, antisocial, agresi- 
vo/sádica, compulsiva, pasivo/agresiva y autodestructiva), tres son escalas graves de personalidad (paranoide, esquizotípica y límite) -que reflejan niveles moderados o altos de deterioro y evalúan el eje II del DSM IV-, seis escalas de síndromes clínicos (ansiedad, histeriforme, hipomanía, distimia, abuso de alcohol y abuso de drogas) -que intentan evaluar trastornos de gravedad moderada-, y tres escalas de síndromes clínicos de gravedad marcada (pensamiento psicótico, depresión mayor y trastorno delirante). Además, el test proporciona información en cuatro escalas adicionales: validez, sinceridad y deseabilidad y alteración.

Millon (1999) establece que una puntuación en la Tasa Base (TB) mayor a 74, en cualquiera de las escalas, indica sospecha de presencia de un trastorno; y una TB mayor a 84 indica presencia y severidad de este. Como señala el autor, el instrumento presenta criterios adecuados de fiabilidad y validez. Las puntuaciones de estabilidad están en torno a ,78 para las escalas de TP y de ,80 para las escalas de síndromes clínicos. En este estudio se ha considerado la presencia de un TP cuando la puntuación en la TB del MCMI-II es superior a 84 , de acuerdo con los criterios más conservadores y exigentes de Wetzler (1990).

El segundo instrumento utilizado es la Entrevista para los Trastornos de Ansiedad (Anxiety Disorder Interview Schedule) según el DSM IV, elaborada por Di Nardo, Brown y Barlow (1994) y traducida y adaptada al castellano por Martín García-Sancho (documento sin publicar), se trata de un instrumento de formato heteroaplicado. A través de este, se realiza una evaluación de los TA y trastornos coocurrentes más comunes, como lo son los del estado de ánimo, abuso y depen- dencia de sustancias, trastornos somatomorfos, entre otros. El formato de aplicación contiene ítems con respuestas dicotómicas (de sí y no), escalas de 0 a 8 puntos (para evaluar la frecuencia e intensidad de la sintomatología) y preguntas abiertas de interés clínico específico para cada TA del DSM IV. Se trata de un instrumento adecuado a nivel de fiabilidad y validez. Presenta una alta confiabilidad test-retest en diversos estudios, que va desde ,68 hasta 1, y $k=, 64$ para correlaciones con entrevistas diagnósticas pares con jueces (Di Nardo, Brown, Lawton, \& Barlow, 1995).

\section{Procedimiento}

Los participantes de este estudio fueron reclutados de la base de datos de la Clínica de Psicología de la Universidad de Granada, situada en la Facultad de Psicología. Son usuarios del programa de Evaluación y Tratamiento de los Trastornos de Ansiedad que se desarrolla allí desde hace 14 años. Concretamente, los participantes de este estudio corresponden a usuarios del programa en los últimos 5 años. En el 90,32\% de los casos, era la primera vez que acudían a un psicólogo.

Este programa está abierto a personas vinculadas con la Universidad de Granada (alumnos/as, personal de administración y servicios, y personal docente e investigador). Cada año, al comienzo de curso, se abre el plazo de solicitud. Los interesados solicitan, de forma voluntaria, ser atendidos en el programa. Inicialmente pasan por una entrevista tipo screening, cuyo objetivo es valorar si se trata de un TA. En esta primera entrevista, se le entrega a cada usuario un documento en el que se le informa que puede formar parte de un estudio y se le solicita que firme el consentimiento informado. No reciben com- 
pensación económica por participar en el estudio. El diseño y aplicación de esta investigación ha seguido fielmente todas las normas éticas elaboradas en la Declaración de Helsinky (Asociación Médica Mundial, 2008).

Posteriormente, los usuarios que son valorados como posibles personas con TA, pasan a una evaluación más específica y pormenorizada, realizada por psicólogos especialistas que colaboran en esta clínica. Se incluye la Entrevista para los Trastornos de Ansiedad, para realizar el diagnóstico siguiendo los planteamientos del DSM IV y el diagnóstico diferencial, instrumentos de autoinforme (entre ellos, el MCMI-II), así como una evaluación psicofisiológica. Una vez realizado el proceso de evaluación, los usuarios pasan a la fase de intervención.

\section{Análisis de datos}

Los resultados fueron analizados utilizando el paquete estadístico SPSS 15.0. En primer lugar, se procedió a comprobar si existían diferencias significativas entre los cuatro grupos (fobia específica, fobia social, trastorno de ansiedad generalizada y trastorno de pánico y agorafobia) en las escalas básicas y escalas graves de los TP. Para ello se realizaron diferentes análisis de varianza (ANOVA, por su nombre en inglés Analysis of Variance), siendo la variable independiente el diagnóstico de TA, con cuatro niveles (fobia específica, fobia social, trastorno de ansiedad generalizada y trastorno de pánico/agorafobia); y las variables dependientes, la puntuación directa en las 10 escalas básicas, las tres escalas graves y las nueve escalas clínicas del MCMI-II.

En segundo lugar, para comparar la frecuencia de la presencia de los TP en los distintos grupos de TA, se utilizó Chi cuadrado $\left(\chi^{2}\right)$ como prueba no paramétrica, usando como criterio de presencia de un $\mathrm{TP}$, el punto de corte de $\mathrm{TB}>84$; es un criterio más exigente y conservador que el que habitualmente se utiliza (TB > 74).

\section{Resultados}

En primer lugar, se procedió a comprobar si existían diferencias significativas entre los cuatro grupos en las puntuaciones directas de las 10 escalas básicas y tres escalas graves de los TP.

Los resultados encontrados muestran la presencia de diferencias estadísticamente significativas entre los grupos en las escalas básicas del MCMI-II esquizoide ( $F[3$, $27]=3,34, p<, 05)$, evitativa, $(F[3,27]=$ $6,12, p<, 05)$, dependiente $(F[3,27]=$ $3,05, p<, 05)$ y autodestructiva $(F[3,27]$ $=6,6, p<, 05)$; y en dos de las escalas graves, la esquizotípica $(F[3,27]=8,53$, $p<, 05)$ y límite $(F[3,27]=3,21, p<$ ,05). Siendo mayores las puntuaciones de los grupos ansiedad generalizada y fobia social en las escalas esquizoide, evitativa, autodestructiva, esquizotípica y límite. Los grupos de ansiedad generalizada y pánico/agorafobia presentan mayores puntuaciones en dependencia (ver tabla 1). En todos los casos, es el grupo de fobia específica el que presenta puntuaciones significativamente más bajas que el resto de los grupos.

Aplicada la prueba ad hoc de Tukey, se observan diferencias significativas $(p<$ ,05) en la escala esquizotípica entre el grupo fobia específica y el grupo de pánico/agorafobia; y en la escala autodestructiva, observamos diferencias significativas entre el grupo fobia específica y los grupos de ansiedad generalizada y pánico/agorafobia. 
Tabla 1

Puntuaciones directas y significación en las escalas del MCMI-II en los cuatro grupos

\begin{tabular}{lccccc}
\hline Grupo & $\begin{array}{c}\text { Fobia } \\
\text { específica }\end{array}$ & Fobia social & $\begin{array}{c}\text { Ansiedad } \\
\text { generalizada }\end{array}$ & $\begin{array}{c}\text { Pánico / } \\
\text { Agorafobia }\end{array}$ & \multirow{2}{*}{$F$} \\
\cline { 2 - 5 } & \multicolumn{2}{c}{$M(D T)$} & \\
\hline Escalas básicas & & & & \\
Esquizoide & $18,00(6,76)$ & $26,75(7,18)$ & $27,83(7,06)$ & $22,00(5,91)$ & $3,34^{*}$ \\
Fóbica/Evitativa & $17,33(7,52)$ & $35,25(12,04)$ & $36,30(12,50)$ & $24,42(8,62)$ & $6,12^{*}$ \\
Dependiente & $27,56(4,65)$ & $31,50(3,32)$ & $39,17(11,43)$ & $35,00(8,31)$ & $3,05^{*}$ \\
Histriónica & $26,33(11,31)$ & $23,25(7,14)$ & $26,67(12,00)$ & $32,58(7,22)$ & 1,36 \\
Narcisista & $30,22(8,80)$ & $23,50(7,00)$ & $29,67(13,25)$ & $36,17(8,69)$ & 2,01 \\
Antisocial & $20,56(4,61)$ & $24,75(7,93)$ & $19,17(7,36)$ & $22,92(6,08)$ & 0,91 \\
Agresivo/Sádica & $28,89(8,88)$ & $30,00(12,46)$ & $25,50(10,84)$ & $30,83(6,71)$ & 0,49 \\
Compulsiva & $36,44(9,00)$ & $41,50(2,89)$ & $38,33(7,79)$ & $39,17(5,15)$ & 0,56 \\
Pasivo/Agresiva & $26,00(10,54)$ & $30,50(17,65)$ & $34,00(14,10)$ & $32,42(8,59)$ & 0,74 \\
Autodestructiva & $14,22(6,63)$ & $30,00(13,12)$ & $37,17(12,78)$ & $27,58(10,20)$ & $6,65^{*}$ \\
Escalas graves & & & & & \\
Esquizotípica & $10,89(5,84)$ & $30,50(8,51)$ & $31,50(13,37)$ & $19,75(7,90)$ & $8,53^{*}$ \\
Límite & $21,11(11,65)$ & $39,25(25,57)$ & $42,67(15,41)$ & $35,00(11,62)$ & $3,21^{*}$ \\
Paranoide & $22,00(8,14)$ & $26,00(1,41)$ & $30,00(12,10)$ & $28,83(8,60)$ & 1,39 \\
\hline
\end{tabular}

Nota: $M=$ Media; $D T=$ Desviación Típica.

$* p<, 05$

En la escala esquizotípica es marginalmente significativa la diferencia entre el grupo de fobia específica y los grupos de fobia social y ansiedad generalizada $(p=, 05)$. Por otra parte, en la escala evitativa se observan diferencias próximas a la significación entre el grupo fobia específica y el grupo ansiedad generalizada $(p=, 06)$.

El análisis de las escalas clínicas nos indica diferencias significativas en la mayoría de estas. Concretamente, puntúa significativamente más bajo en las escalas de ansiedad $(F[3,27]=9,34, p<, 05)$, histeriforme $(F[3,27]=8,07, p<, 05)$, hipomanía $(F[3,27]=6,50, p<, 05)$, neurosis depresiva $(F[3,27]=6,69, p<, 05)$, abuso de alcohol $(F[3,27]=6,84, p<, 05)$, pensamiento psicótico $(F[3,27]=6,95, p$ $<, 05)$ y depresión mayor $(F[3,27]=$ $5,03, p<, 05)$.
Aplicada la prueba ad hoc de Tukey, se observa que en todos estos casos el grupo de fobia específica presenta una puntuación significativamente más baja que el resto de los grupos (todos los $p s<, 05$ ).

No se observan diferencias significativas entre los grupos en las escalas de abuso de drogas $(p=, 13)$ y delirios psicóticos $(p=, 12)$.

En relación a los trastornos que se dan con más frecuencia en el grupo de personas con TA, podemos observar que las escalas más frecuentes son: compulsiva $(41,94 \%)$, esquizoide $(29,03 \%)$, dependiente $(25,81 \%)$ y esquizotípica (22,58\%). El porcentaje de participantes con al menos un TP es del $74,20 \%$. Los TP que no aparecen, o aparecen mínimamente, en este grupo de personas diagnosticadas con TA, son el 
trastorno límite, el trastorno antisocial y el trastorno pasivo-agresivo.

Los resultados de la prueba no paramétrica Chi cuadrado aplicada indican que existen diferencias significativas entre los grupos en las escalas evitativa $\left(\chi^{2}=\right.$ $10,24, p<, 05)$, dependiente $\left(\chi^{2}=10,11\right.$, $p<, 05)$ y esquizotípica $\left(\chi^{2}=9,55, p<\right.$ ,05); y próximas al nivel de significación, las escalas esquizoide $\left(\chi^{2}=7,27, p=, 06\right)$ $\mathrm{y}$ autodestructiva $\left(\chi^{2}=7,59, p=, 06\right)$; destacando el porcentaje de fóbicos sociales en la escala evitativa, esquizoide y esquizotípica, el porcentaje de ansiedad generalizada en las escalas esquizoide, esquizotípica y autodestructiva, y el porcentaje de pánico y agorafobia en la escala de dependencia (ver tabla 2).
Finalmente, para el análisis de diagnósticos múltiples en cada trastorno, hemos realizado, en primer lugar, un análisis de prueba no paramétrica Chi cuadrado. Como podemos observar en la tabla 3, los resultados no son significativos ( $p=$ ,09).

En segundo lugar, se decidió agrupar a los participantes en tres grupos (ningún TP, un TP, dos o más TP). En este caso, las diferencias sí son significativas, siendo el grupo de fobia específica el que presenta mayor porcentaje de participantes sin ningún TP. Por su parte, los grupos de ansiedad generalizada, fobia social y pánico, presentaron un mayor porcentaje de TP (en ese orden, ver tabla 4).

Tabla 2

Número de sujetos $y \%$ con puntuaciones $T B \geq 84$ en las escalas del MCMI-II

\begin{tabular}{|c|c|c|c|c|c|c|}
\hline \multirow[t]{2}{*}{ Grupo } & $\begin{array}{c}\text { Fobia } \\
\text { específica }\end{array}$ & $\begin{array}{l}\text { Fobia } \\
\text { social } \\
\end{array}$ & $\begin{array}{c}\text { Ansiedad } \\
\text { generalizada }\end{array}$ & $\begin{array}{c}\text { Pánico / } \\
\text { agorafobia }\end{array}$ & $\begin{array}{r}\text { Todos } \\
\text { los TA } \\
\end{array}$ & \multirow[t]{2}{*}{$\chi^{2}$} \\
\hline & \multicolumn{5}{|c|}{$n(\%)$} & \\
\hline \multicolumn{7}{|l|}{ Escalas básicas } \\
\hline Esquizoide & $1(11,11)$ & $2(50,00)$ & $4(66,67)$ & $2(16,67)$ & $9(29,03)$ & 7,27 \\
\hline Fóbica/Evitativa & $0(0)$ & $2(50,00)$ & $2(33,33)$ & $0(0)$ & $4(12,90)$ & $10,24 *$ \\
\hline Dependiente & $0(0)$ & $0(0)$ & $4(66,67)$ & $4(33,00)$ & $8(25,81)$ & $10,11 *$ \\
\hline Histriónica & $2(22,22)$ & $0(0)$ & $1(16,67)$ & $3(25,00)$ & $6(19,35)$ & 1,28 \\
\hline Narcisista & $1(11,11)$ & $0(0)$ & $1(16,67)$ & $3(25,00)$ & $5(16,13)$ & 1,64 \\
\hline Antisocial & $0(0)$ & $1(25,00)$ & $0(0)$ & $0(0)$ & $1(3,23)$ & 6,98 \\
\hline Agresivo/Sádica & $1(11,11)$ & $1(25,00)$ & $0(0)$ & $1(8,33)$ & $3(9,68)$ & 1,76 \\
\hline Compulsiva & $3(33,33)$ & $2(50,00)$ & $3(50,00)$ & $5(41,67)$ & $13(41,94)$ & 0,54 \\
\hline Pasivo/Agresiva & $0(0)$ & $1(25,00)$ & $0(0)$ & $0(0)$ & $1(3,23)$ & 4,77 \\
\hline Autodestructiva & $0(0)$ & $1(25,00)$ & $3(50,00)$ & $1(8,33)$ & $5(16,13)$ & 7,59 \\
\hline \multicolumn{7}{|l|}{ Escalas graves } \\
\hline Esquizotípica & $0(0)$ & $3(75,00)$ & $2(33,33)$ & $2(16,67)$ & $7(22,58)$ & $9,55^{*}$ \\
\hline Límite & $0(0)$ & $0(0)$ & $0(0)$ & $0(0)$ & $0(0)$ & 0 \\
\hline Paranoide & $0(0)$ & $0(0)$ & $2(33,33)$ & $1(8,33)$ & $3(9,68)$ & 5,26 \\
\hline Total de TP & 8 & 13 & 22 & 22 & 65 & \\
\hline
\end{tabular}

Nota: $n=$ número de sujetos que presentan puntuaciones $\mathrm{TB} \geq 84 ; \%=$ porcentaje de sujetos que presentan puntuaciones $\mathrm{TB} \geq 84$; Todos $\operatorname{los} \mathrm{TA}=$ suma de sujetos con TA en cada una de las escalas.

$* p<, 05$ 
Tabla 3

Número de trastornos de personalidad en los cuatro grupos

\begin{tabular}{lcccccc}
\hline \multirow{2}{*}{ Grupo } & $\begin{array}{c}\text { Fobia } \\
\text { específica }\end{array}$ & Fobia social & $\begin{array}{c}\text { Ansiedad } \\
\text { generalizada }\end{array}$ & $\begin{array}{c}\text { Pánico / } \\
\text { agorafobia }\end{array}$ & Total & \multirow{5}{*}{$\chi^{2}$} \\
\cline { 2 - 5 } & \multicolumn{5}{c}{$M(D T)$} \\
\hline
\end{tabular}

Nota: $M=$ Media; $D T=$ Desviación Típica.

Tabla 4

Porcentaje de trastornos de personalidad en los cuatro grupos

\begin{tabular}{lccccc}
\hline Grupo & $\begin{array}{c}\text { Fobia } \\
\text { específica }\end{array}$ & Fobia social & $\begin{array}{c}\text { Ansiedad } \\
\text { generalizada }\end{array}$ & $\begin{array}{c}\text { Pánico / } \\
\text { agorafobia }\end{array}$ & $\chi^{2}$ \\
\hline Ningún TP & $55,56 \%$ & $25,00 \%$ & $0 \%$ & $16,67 \%$ & \\
Un TP & $22,22 \%$ & $0 \%$ & $0 \%$ & $33,33 \%$ & $12,54 *$ \\
Dos o más TP & $22,22 \%$ & $75,00 \%$ & $100 \%$ & $50,00 \%$ & \\
\hline
\end{tabular}

$* p<, 05$

\section{Discusión}

El objetivo del estudio ha sido explorar, de manera descriptiva, la coocurrencia entre los TP y los TA. Ha sido de interés explorar, no solo la coocurrencia entre los TA en general y los TP, sino las diferencias entre los distintos TA y los perfiles de TP.

Se encontró que en los participantes con TA, los TP que se observan con más frecuencia son personalidad compulsiva, esquizoide, dependiente y esquizotípica. Los datos coinciden solo en parte con los obtenidos por Flick et al. (1993) con pacientes ambulatorios, en los que encontraron que los TP más habituales fueron personalidad tipo evitativa, paranoide, límite y obsesivo-compulsiva. Es posible que los resultados sean diferentes a los obtenidos en el presente estudio, debido al instrumento de evaluación utilizado (dichos autores utilizaron la Entrevista Clínica Estructurada para el Eje II del DSM III-R (Structured Clinical Interview for DSM).
En este estudio se observa, además, diferencias significativas entre los grupos de TA en las puntuaciones directas en las escalas evitativa, esquizoide, autodestructiva, esquizotípica y límite; siendo los grupos de ansiedad generaliza y fobia social los que presentan puntuaciones significativamente más altas, resultados que van en la misma línea de los presentados por Reich (2009).

En la escala de personalidad dependiente también se encontraron diferencias significativas entre los grupos, siendo los grupos de ansiedad generalizada y trastorno de pánico y agorafobia los que presentan puntuaciones más altas. En lo referente al trastorno de pánico y agorafobia, nuestros datos van en la línea de los encontrados en diversas investigaciones (Albert et al., 2006; Marchesi et al., 2006; Starcevic et al., 2008; Telch et al., 2011).

El porcentaje de participantes que presentan al menos un TP, es de 74,2\%. Destaca este porcentaje sobre el que presenta el estudio de Dyck et al. (2001). Concreta- 
mente, en esta investigación se encuentra que el $24 \%$ de los pacientes evaluados, presentan al menos un TP coocurrente con el diagnóstico de TA. Creemos que una posible explicación podría ser el instrumento de evaluación utilizado; mientras que Dyck et al. (2001) utilizan la entrevista clínica estructurada, en el presente estudio se ha utilizado el MCMIII, instrumento específicamente diseñado para evaluar este tipo de trastornos; además, se ha utilizado un criterio estricto (TB $\geq 84)$. En este sentido, Friborg et al. (2014) han encontrado que cuando se utiliza un cuestionario para evaluar los TP, la comorbilidad es mucho mayor.

Analizadas las diferencias en el porcentaje de pacientes con TP en cada grupo, observamos que existen diferencias significativas en las escalas evitativa (mayor porcentaje en el grupo de fobia social), esquizotípica (mayor porcentaje en fobia social), esquizoide (mayor en ansiedad generalizada y fobia social), dependiente (en ansiedad generalizada y pánico y agorafobia) y autodestructiva (mayor en ansiedad generalizada); lo que indica perfiles concretos de personalidad, en función del TA concreto.

En cuanto al diagnóstico múltiple, son los grupos de ansiedad generalizada y fobia social los que presentan la mayor frecuencia de diagnósticos múltiples (y por tanto, mayor gravedad; según los resultados obtenidos por Dolan et al., 1995). Por su parte, es el grupo de fobia específica el que presenta significativamente un menor porcentaje de diagnósticos múltiples y un mayor porcentaje de personas sin ningún TP.

Los datos obtenidos en relación al grupo trastorno de pánico y agorafobia, indican que el perfil de TP sería: dependientes y compulsivos, trastornos pertenecientes al grupo o cluster C del DSM V (personalidad por evitación, personalidad dependiente y personalidad obsesivo-compulsiva; APA, 2013) y narcisista.

Los presentes datos sobre dependencia coinciden con los publicados en numerosos estudios (Albert et al., 2006; Marchesi et al., 2006; Osma et al., 2014a; Starcevic et al., 2008; Telch et al., 2011). Estos mismos autores recogen que la personalidad evitativa también es característica del pánico y agorafobia, dato que en nuestro estudio no se ha obtenido. Diferencias en el instrumento utilizado y criterio de inclusión de los sujetos (TB > 84, en el presente estudio), podrían explicar parte de las diferencias observadas.

Concretamente, en el estudio de Osma et al. (2014b) los autores obtienen algunas diferencias con respecto a la presente investigación, no solo en las escalas que son más significativas, sino incluso en las puntuaciones medias obtenidas en todas las escalas (en el estudio de Osma et al., 2014b, las puntuaciones directas son más altas). Consideramos que estas diferencias pueden deberse a que el grupo de Osma utiliza el MCMI-III y nosotros hemos utilizado el MCMI-II. Además, otra diferencia que podemos observar, es que la muestra de Osma estuvo compuesta por un 50\% de hombres y un 50\% de mujeres, mientras que en nuestro estudio, las mujeres constituyen el $83,87 \%$ de la muestra evaluada.

Los resultados obtenidos respecto a la identificación de grupos y TP específicos coinciden, en parte, con el estudio de metanálisis recientemente publicado por Friborg et al. (2013). Estos autores concluyen que el grupo $\mathrm{C}$ de los TP es el que mayor proporción obtiene en muestras de TA en general y, específicamente, en tras- 
tornos de fobia social (sobre todo, la escala evitativa), seguido del grupo B y del A.

Los datos obtenidos en el grupo de ansiedad generalizada nos indican que es el grupo de TA con mayor tasa de diagnósticos múltiples (indicativo de gravedad), cuyo perfil de TP sería: esquizoide, dependiente, autodestructiva, evitativa y compulsiva. Esta última escala de personalidad (compulsiva), si bien no se observan diferencias significativas entre los grupos, ha resultado ser una de las escalas con mayor intensidad y frecuencias en los cuatro trastornos estudiados; datos que van en la línea de los encontrados por diversos investigadores (Albert et al., 2006; Marchesi et al., 2006; Osma et al., 2014a; Starcevic et al., 2008; Telchet al., 2011).

Los resultados obtenidos en el grupo de fobia social indican que es el segundo grupo de trastornos con mayor tasa de TP y cuyo perfil sería: personalidad esquizoide, esquizotípica, compulsiva y evitativa. Esta última escala coincide con los datos publicados sobre fobia social (Feske et al., 1996) y, parcialmente, con los datos obtenidos por Dyck et al. (2001).

Observamos que los grupos de ansiedad generalizada y fobia social, son los que presentan los rasgos de personalidad más disfuncionales. Dado que el presente estudio es básicamente descriptivo, y entendemos que existe una serie de limitaciones (que se recogen al final de este apartado), sería interesante abordar en una segunda fase de esta investigación otros elementos que ayuden a clarificar estos resultados.

Los datos obtenidos para el grupo de fobia específica, claramente indican que se trata del grupo con menor coocurrencia con TP, tanto a nivel de intensidad como de frecuencia de personas con TP. Ade- más, los resultados sobre diagnósticos múltiples nos indican que se trata del grupo de menor gravedad, tal como ha sido propuesto en la literatura sobre este trastorno (Belloch et al., 2008).

En el análisis de las escalas clínicas del MCMI-II, se observan puntuaciones significativamente más bajas en el grupo de fobia específica en las escalas de ansiedad, histeriforme, hipomanía, neurosis depresiva, abuso de alcohol, pensamiento psicótico y depresión mayor. Estos datos son coherentes con los obtenidos en relación a las puntuaciones en los distintos $\mathrm{TP}$, así como en relación al número de diagnósticos.

El grupo de fobia específica presenta menor complejidad en los diagnósticos, menor psicopatología general y menor coocurrencia de trastornos; entendemos, por tanto, que menor gravedad. Nuestros resultados van en la línea de lo propuesto por Belloch et al. (2008), pero no son coherentes con los obtenidos por Stinson et al. (2007). En relación a los datos que estos investigadores proponen dentro de las fobias específicas, se podrían distinguir dos tipos: una fobia específica más concreta, en la que el miedo está muy localizado en un estímulo fóbico (y por tanto, produciría menos interferencia); y otro tipo de fobia específica, más generalizada, que produciría mayor incapacidad $\mathrm{y}$ deterioro en el funcionamiento.

Los resultados de este estudio indican la relevancia de evaluar de forma adecuada y sistemática el eje I y II, es decir, los síndromes clínicos y los TP. La coocurrencia de TA y TP va a condicionar la intervención a desarrollar con un paciente, la tasa de abandono, así como el pronóstico (Friborg et al., 2013; NewtonHowes et al., 2010; Ozkan \& Altindag, 2005; Reich, 2003). 
El presente estudio presenta limitaciones que es necesario destacar. El pequeño tamaño de la muestra de los diferentes grupos, así como las diferencias en el número de participantes en cada uno de estos, restan potencia a los análisis estadísticos, por lo que se sugiere cautela en la generalización de los resultados.

Sería interesante, además de aumentar el número de sujetos por grupo, incluir otros TA, así como la posibilidad de distinguir entre trastorno de pánico y trastorno de agorafobia como dos grupos independientes.

Por otra parte, el nivel sociocultural de la muestra utilizada no representa al resto de la población, ya que en su mayoría son estudiantes universitarios, personal de administración y servicios, y personal docente e investigador; un nivel de estudios muy alto. Sería recomendable ampliar el número de participantes a otros sectores de la población.

Por último, sería interesante evaluar los TP a través de entrevistas específicas y no solo mediante cuestionarios, dado que los datos parecen indicar que la coocurrencia es mayor cuando se utilizan estos instrumentos.

Es importante mencionar que la presente investigación constituye un estudio preliminar, que aborda un importante tópico en el ámbito de la psicología clínica, cuyos resultados deben tomarse con cautela, y cuyas consecuencias son de gran relevancia para entender la compleja relación entre los síndromes clínicos y los TP, y los resultados que se obtienen en el ámbito aplicado.

\section{Referencias}

Albert, U., Maina, G., Bergessio, C., \& Bogetto, F. (2006). Axis I and II comorbidities in subjects with and without nocturnal panic. Depression and Anxiety, 23(7), 422-428. http://dx.doi.org/10.1002/da.20200

American Psychiatric Association. (1980). Diagnostic and statistical manual of mental disorders DSM-III. Washington, DC: American Psychiatric Association.

American Psychiatric Association. (1994). Diagnostic and statistical manual of mental disorders DSM-IV. Whashington, DC: American Psychiatric Association.

American Psychiatric Association. (2013). Guía de consulta de los criterios diagnósticos del DSM-5. Madrid, España: Editorial Médica Panamericana.

Aragona, M. (2009). The role of comorbidity in the crisis of the current psychiatric classification system. Philosophy, Psychiatry, \& Psychology, 16(1), 1-11

http://dx.doi.org/10.1353/ppp.0.0211

Asociación Médica Mundial. (2008). Declaración de Helsinki: principios éticos para las investigaciones médicas en seres humanos. Recuperado de http://www.wma.net/es/30publications/10policies/b3/index.html

Belloch, A., Sandín, B., \& Ramos, F. (2008) Manual de Psicopatología (Vol. 1). Madrid, España: McGraw-Hill. 
Bienvenu, O. J., Stein, M. B., Samuels, J. F., Onyike, C. U., Eaton, W. W., \& Nestadt, G. (2009). Personality disorder traits as predictors of subsequent first-onset panic disorder or agoraphobia. Comprehensive Psychiatry, 50(3), 209-214.

http://dx.doi.org/10.1016/j.comppsych.2008.08.006

Brown, T. A. \& Barlow, D. H. (2009). A proposal for a dimensional classification system based on the shared features of the DSMI-IV anxiety and mood disorders: Implications for assessment and treatment. Psychological Assessment, 21(3), 256-271. http://dx.doi.org/10.1037/a0016608\%20

Di Nardo, P. A., Brown, T. A., \& Barlow, D. H. (1994). Anxiety Disorders Interview Schedule for DSM-IV: Lifetime version (ADIS-IV-L). San Antonio, Texas: Psychological Corporation.

Di Nardo, P. A., Brown, T. A., Lawton, J. K., \& Barlow, D. H. (noviembre, 1995). The Anxiety Disorders Interview Schedule for DSM-IV Lifetime Version: Description and initial evidence for diagnostic reliability. Artículo presentado en la $29^{\text {th }}$ Annual Meeting of the Association for Advancement of Behavior Therapy, Washington, District of Columbia.

Dolan, B., Evans, C., \& Norton, K. (1995). Multiple axis-II diagnoses of personality disorder. British Journal of Psychiatry, 166(1), 107-112.

http://dx.doi.org/10.1192/bjp.166.1.107

Dunayevich, E., Sax, K. W., Keck, P. E. Jr., McElroy, S. L., Sorter, M. T., McConville, B. J., \& Strakowski, S. M. (2000). Twelve-month outcome in bipolar patients with and without personality disorders. Journal of Clinical Psychiatry, 61(2), 134-139.

http://dx.doi.org/10.4088/jcp.v61n0209

Dyck, I. R., Phillips, K. A., Warshaw, M. G., Dolan, R. T., Shea, M. T., Stout, R. L., ... Keller, M. B. (2001). Patterns of personality pathology in patients with generalized anxiety disorder, panic disorder with and without agoraphobia, and social phobia. Journal of Personality Disorders, 15(1), 60-71. Recuperado de http://www.ncbi.nlm.nih.gov/pubmed/11236815

Fernández Montalvo, J. \& Echeburúa, E. (2006). Juego patológico y trastornos de personalidad: un estudio piloto con el MCMI-II. Psicothema, 18(3), 453-458. Recuperado de http://www.psicothema.com/pdf/3237.pdf

Feske, U., Perry, K. J., Chambless, D. L., Renneberg, B., \& Goldstein, A. J. (1996). Avoidant personality disorder as a predictor for treatment outcome among generalized social phobics. Journal of Personality Disorders, 10(2), 174-184.

http://dx.doi.org/10.1521/pedi.1996.10.2.174

Flick, S. N., Roy-Byrne, P. P., Cowley, D. S., Shores, M. M., \& Dunner, D. L. (1993). DSM-III-R personality disorders in a mood and anxiety disorders clinic: Prevalence, comorbidity, and clinical correlates. Journal of Affective Disorders, 27(2), 71-79. http://dx.doi.org/10.1016/0165-0327(93)90079-Y

Friborg, O., Martinsen, E. W., Martinussen, M., Kaiser, S., Øvergård, K. T., \& Rosenvinge, J. H. (2014). Comorbidity of personality disorders in mood disorders: A meta-analytic review of 122 studies from 1988 to 2010. Journal of Afecttive Disorders, 152-154, 1-11. http://dx.doi.org/10.1016/j.jad.2013.08.023 
Friborg, O., Martinussen, M., Kaiser, S., Øvergård, K. T., \& Rosenvinge, J. H. (2013). Comorbidity of personality disorders in anxiety disorders: A meta-analysis of 30 years of research. Journal of Affective Disorders, 145(2), 143-155.

http://dx.doi.org/10.1016/j.jad.2012.07.004

Grant, B. F., Hasin, D. S., Blanco, C., Stinson, F. S., Chou, S. P., Goldstein, R. B, ... Huang, B. (2005). The epidemiology of social anxiety disorder in the United States: Results from the National Epidemiologic Survey on Alcohol and Related Conditions. Journal of Clinical Psychiatry, 66(11), 1351-1361.

http://dx.doi.org/10.4088/jcp.v66n1102

Gunderson, J. G. \& Gabbard, G. O. (Ed.) (2000). Psychotherapy for personality disorders. Washington, District of Columbia: American Psychiatric Press.

Jakovljević, M. \& Crnčević, Z. (2012). Comorbidity as an epistemological challenge to modern psychiatry. Dialogues in Philosophy, Mental and Neuro Sciences, 5(1), 1-13. Recuperado de http://www.crossingdialogues.com/Ms-A12-07.pdf

Kotov, R., Gámez, W., Schmidt, F., \& Watson, D. (2010). Linking “big” personality traits to anxiety, depressive, and substance use disorders: A meta-analysis. Psychological Bulletin, 136(5), 768-821.

http://dx.doi.org/10.1037/a0020327

López Durán, A. \& Becoña Iglesias, E. (2006). Patrones y trastornos de personalidad en personas con dependencia de la cocaína en tratamiento. Psicothema, 18(3), 578-583. Recuperado de

http://www.psicothema.com/pdf/3256.pdf

Marchesi, C., Cantoni, A., Fontò, S., Giannelli, M. R., \& Maggini, C. (2005). The effect of pharmacotherapy on personality disorders in panic disorder: A one year naturalistic study. Journal of Affective Disorders, 89(1-3), 189-194.

http://dx.doi.org/10.1016/j.jad.2005.07.007

Marchesi, C., De Panfilis, C., Cantoni, A., Fontò, S., Giannelli, M. R., \& Maggini, C. (2006). Personality disorders and response to medication treatment in panic disorder: A 1-year naturalistic study. Progress in Neuro-Psychopharmacology and Biological Psychiatry, 30(7), 1240-1245.

http://dx.doi.org/10.1016/j.pnpbp.2006.03.010

Markovitz, P. J. (2004). Recent trends in the pharmacotherapy of personality disorders. Journal of Personality Disorders, 18(1), 90-101. http://dx.doi.org/10.1521/pedi.18.1.90.32768

Martín García-Sancho, J. C. Entrevista Diagnóstica de Trastornos por ansiedad según el DSM-IV: Versión L (ADIS-IV-L) (documento sin publicar).

Martino, F., Menchetti, M., Pozzi, E., \& Berardi, D. (2012). Predictors of dropout among personality disorders in a specialist outpatients psychosocial treatment: A preliminary study. Psychiatry and Clinical Neurosciences, 66(3), 180-186.

http://dx.doi.org/10.1111/j.1440-1819.2012.02329.x 
Millon, T. (1999). MCMI-II. Inventario Clínico Multiaxial de Millon-II. Manual. Madrid, España: TEA Ediciones.

Newton-Howes, G., Tyrer, P., Anagnostakis, K., Cooper, S., Bowden-Jones, O., \& Weaver, T. (2010). The prevalence of personality disorder, its comorbidity with mental state disorders, and its clinical significance in community mental health teams. Social Psychiatry and Psychiatric Epidemiology, 45(4), 453-460.

http://dx.doi.org/10.1007/s00127-009-0084-7

Oldham, J. M., Skodol, A. E., Kellman, H. D., Hyler, S. E., Rosnick, L., \& Davies, M. (1992). Diagnosis of DSM III-R personality disorders by two structured interviews: Patterns of comorbidity. American Journal of Psychiatry, 149(2), 213-220.

http://dx.doi.org/10.1176/ajp.149.2.213

Osma, J., García-Palacios, A., Botella, C., \& Barrada, J. R. (2014a). Personalidad y sensibilidad a la ansiedad como factores de vulnerabilidad en el trastorno de pánico. Behavioral Psychology / Psicología Conductual, 22(2), 185-201. Recuperado de http://www.unizar.es/barrada/papers/anxiety_sensibility.pdf

Osma, J., García-Palacios, A., Botella, C., \& Barrada, J. R. (2014b). Personality disorders among patients with panic disorder and individuals with high anxiety sensitivity. Psicothema, 26(2), 159-165.

http://dx.doi.org/10.7334/psicothema2013.248

Ozkan, M. \& Altindag, A. (2005). Comorbid personality disorders in subjects with panic disorder: Do personality disorders increase clinical severity? Comprehensive Psychiatry, 46(1), 20-26.

http://dx.doi.org/10.1016/j.comppsych.2004.07.015

Prieto Cuéllar, M., Vera Guerrero, M. N., Pérez Marfil, M. N., \& Ramírez Uclés, I. (2007). Cronicidad de los trastornos del estado de ánimo: relaciones con actitudes cognitivas disfuncionales y con alteraciones de la personalidad. Clínica y Salud, 18(2), 203-219. Recuperado de

http://scielo.isciii.es/pdf/clinsa/v18n2/v18n2a05.pdf

Reich, J. (2003). The effect of axis II disorders on the outcome of treatment of anxiety and unipolar depressive disorders: A review. Journal of Personality Disorders, 17(5), 387-405. http://dx.doi.org/10.1521/pedi.17.5.387.22972

Reich, J. (2009). Avoidant personality disorder and its relationship to social phobia. Current Psychiatry Reports, 11(1), 89-93.

http://dx.doi.org/10.1007/s11920-009-0014-0

Rossi, A., Marinangeli, M. G., Butti, G., Scinto, A., Di Cicco, L., Kalyvoka, A., \& Petruzzi, C. (2001). Personality disorders in bipolar and depressive disorders. Journal of Affective Disorders, 65(1), 3-8. Recuperado de http://www.ncbi.nlm.nih.gov/pubmed/11426507

Salavera, C., Puyuelo, M., Tricás, J. M., \& Lucha, O. (2009). Comorbilidad de trastornos de personalidad: estudio en personas sin hogar. Universitas Psychologica, 9(2), 457-467. Recuperado de http://pepsic.bvsalud.org/scielo.php?pid=S1657-92672009000200013\&script=sci_arttext 
Sanderson, W. C., Beck, A. T., \& McGinn, L. K. (1994). Cognitive therapy for generalized anxiety disorder: Significance of comorbid personality disorders. Journal of Cognitive Psychotherapy, 8(1), 13-18. Recuperado de http://www.ingentaconnect.com/content/springer/jcogp/1994/00000008/00000001/art00003

Sandoval, L. X. \& Villamil, V. (1999). La comorbilidad de los trastornos depresivos y de los trastornos de la personalidad. Salud Mental V, 22(3), 34-40. Recuperado de http://inprf-cd.gob.mx/pdf/sm2203/sm220334.pdf

Soeteman, D. I., Hakkaart-van Roijen, L., Verheul, R., \& Busschbach, J. J. (2008). The economic burden of personality disorders in mental health care. Journal of Clinical Psychiatry, 69(2), 259-265. Recuperado de

http://www.ncbi.nlm.nih.gov/pubmed/18363454

Starcevic, V., Latas, M., Kolar, D., Vucinic-Latas, D., Bogojevic, G., \& Milovanovic, S. (2008). Co-occurrence of axis I and axis II disorders in female and male patients with panic disorder with agoraphobia. Comprehensive Psychiatry, 49(6), 537-543.

http://dx.doi.org/10.1016/j.comppsych.2008.02.009

Stinson, F. S., Dawson, D. A., Chou, S. P, Smith, S., Goldstein, R. B., Ruan, W. J., \& Grant, B. F. (2007). The epidemiology of DSM-IV specific phobia in the USA: Results from the National Epidemiologic Survey on Alcohol and Related Conditions. Psychological Medicine, 37(7), 1047-1059.

http://dx.doi.org/10.1017/S0033291707000086

Telch, M. J., Kamphuis, J. H., \& Schmidt, N. B. (2011). The effects of comorbid personality disorders on cognitive behavioral treatment for panic disorder. Journal of Psychiatric Research, 45(4), 469-474.

http://dx.doi.org/10.1016/j.jpsychires.2010.08.008

Wetterborg, D., Långström, N., Andersson, G., \& Enebrink, P. (2015). Borderline personality disorder: Prevalence and psychiatric comorbidity among male offenders on probation in Sweden. Comprehensive Psychiatry, 62, 63-70.

http://dx.doi.org/10.1016/j.comppsych.2015.06.014

Wetzler, S. (1990). The Million Clinical Multiaxial Inventory (MCMI): A review. Journal of Personality Assessment, 55(3-4), 445-464.

http://dx.doi.org/10.1080/00223891.1990.9674083

Zimmerman, M., Rothschild, L., \& Chelminski, I. (2005). The prevalence of DSM-IV personality disorders in psychiatric outpatients. American Journal of Psychiatry, 162(10), 1911-1918. Recuperado de http://dx.doi.org/10.1176/appi.ajp.162.10.1911

Fecha de recepción: 30 de diciembre de 2015

Fecha de aceptación: 7 de junio de 2016 